\title{
Transmission Line Performance Assessment and Design using MATLAB
}

\author{
Aaron Don M. Africa, Jamie Lynn T. Chua, Harold John T. Tan \\ Department of Electronics and Communications Engineering \\ De La Salle University, Manila \\ 2401 Taft Ave., Malate, Manila 1004, \\ Philippines, aaron.africa@dlsu.edu.ph
}

\begin{abstract}
Due to the increase in demand for electrical power yet issues with economic constraints, the efficiency of transmission lines has become a topic of interest for many. In this study, the researchers will be executing a program that actively simulates the transmission line performance test for the short, medium, and long transmission line cases through the use of a numerical computing environment, MATLAB. The parameters of the transmission lines to be assessed will be defined by the user, including aspects such as the length and characteristics of the material. While the program is taking into consideration the individual $\mathrm{ABCD}$ constants of the different length transmission lines for the calculations, the output from this would be observed as the characteristics (e.g. power factor, transmission line efficiency, and voltage regulation) of the user-input transmission line. With specifications on voltage regulation and line transmission efficiency, the results will be analyzed by the system in order to determine whether or not the transmission line being tested by the user is applicable for use.
\end{abstract}

Key words: Transmission Line, Voltage Regulation, Line Efficiency, ABCD Parameters.

\section{INTRODUCTION}

Discovery and enhancement in the various forms of science and technology have in turn resulted in the pressure for manufacturers and developers to produce materials or devices that can do more in a smaller, more efficient form. As such, the demand for electrical power would spike by a significant amount each time something is released given that the production of these devices as well as their function would require a source of energy to operate [1]. Similarly, electrical power is known to become more in-demand during the months of April to May in the Philippines due to weather and temperatures rising. In 2019, Luzon alone had a reading of over 11,000MW in May. Overseen by the Department of Energy, the National Grid Corporation of the Philippines must ensure the consistent supply of power in order to avoid dealing with outages and emergencies [2]. As such, in order to avoid the deficiency and instability of the energy being delivered, there is a need to maintain and upgrade the many connections found between the power supply and the receiving devices, becoming more complex over time. One of the most well-known methods to deal with the safe supply of energy is through the use of transmission lines and associated parts like electrical towers. Generally, these lines are used in areas like radio-frequency, water and gas infrastructure, and transmission of electrical power, wherein the current and voltage pass through the conductor found in the length of the cable [3]. It works by simply allowing the load to flow through its length, thereby providing a means of observation and assessment for the performance of the transmission line. Factors that can affect this include weather conditions and the material used, as well as the length and load being delivered [4]. The analysis of this often serves as the main basis for identifying the operation state, type including the properties, and length of the transmission line that would be the best fit for the system being considered. Parameters which include but are not limited to power factor, voltage regulation, and transmission line efficiency are considered when determining the overall effectiveness of the transmission line [5].

\section{BACKGROUND OF THE STUDY}

As the demand for electrical power increases significantly each year, researchers have been conducting various studies that not only monitor and identify the various transmission lines that best fit a certain type of use, but also discover new methods that can enhance these lines in the most economical and efficient standpoint. One of these alternative techniques that have been identified for optimizing the capacity of the lines included a study that used uprating the voltage to $230 \mathrm{kV}$ flowing in the transmission line [6]. Another study considered the loss in the length of the transmission line, particularly the long one, and proposed an HVAC line with a tuned high-voltage $\mathrm{AC}$ that increased overall power transmission [7]. Overload of the transmission line has also been one of the prominent issues that have yet to be fully overcome, which serves as the baseline of Alminhana et. al's discussion on nonlinear dynamic cascading of the lines. Implementing a time-domain analysis, the transmission line is given parameters that would determine the different failure 
scenarios [8]. Through the applications developed by power companies and referencing previous research from different sources, these approaches can and should be simulated thoroughly before mass implementation. A study that had tackled transmission line analysis was conducted through the use of power charts as a means for basis and consideration, thereby determining the potential mathematical models to be implemented in solving for the actual performance of the line [9]. Taking into consideration the transmission line towers, a study conducted by Balagopal et. al identified a bolted connection model that allowed for the stiffness found in the axial and rotational directions for a better analysis of the power flow [10]. With the presented studies as the general basis and guide, the researchers were able to determine the parameters to consider and result in for the transmission line assessment to be conducted through MATLAB.

\section{STATEMENT OF THE PROBLEM}

In order to deliver electrical power to machines and devices that need it, the most common solution in response to this would be to utilize more transmission lines. However, due to the significant economic and space constraints, this would pose with regards to conditions in most areas, the need to deliver power more efficiently has then been evaluated by alternative methods [11]. For the most part, however, the pressing need to identify and define the parameters to be implemented in the transmission lines in order to properly and effectively apply this to the systems available at present. This tends to become the first step before considering more complicated techniques and approaches for enhancement [12]. With that, this study will be executing the performance line test for transmission lines of varying lengths in order to identify the general bases for the effective delivery of power, and overall determining whether the parameters inputted are acceptable.

\section{SIGNIFICANCE OF THE STUDY}

As the world continues to surpass the boundaries of science and technology, the demand for electrical energy in order to power these discoveries grows exponentially. Not only do the tools and development of these inventions require vast amounts of energy, but also the manufacturing or production processes. Similarly, as the population continues to grow, the pressure increases on energy companies to supply the masses becomes more complicated [13]. As such, the solution that most commonly is brought up is the addition of transmission lines in order to allow more energy to flow to communities and areas that need it. However, the economic limitations of the company versus the people hinder the ability to implement new lines. Furthermore, the countries, where underground power lines have become the norm, have space constraints in terms of the number of wires they can pass through [14]. Consequently, the need to develop new or alternative methods to increase or enhance the capacity of the presently available transmission lines has become a great area of interest [15]. Many researchers have begun to identify new techniques that establish better power flow while also implementing outside parameters such as geological and terrestrial factors; however, understanding transmission lines and their capabilities in terms of length and the material of the wire is the first and most important aspect that should be considered before presenting approaches that overcome these present limitations [16].

\section{DESCRIPTION OF THE SYSTEM}

The system will simulate whether the transmission line is capable of real-life applications depending on the voltage regulation and transmission line efficiency outputs. The system will process the input parameters assigned by the user, then performs its intensive calculations for the output parameters. Depending on the voltage regulation and transmission line efficiency outputs, the program will comment on whether the transmission line will run smoothly or not by commenting Applicable or Not Applicable. In addition, the program will also comment on the type of transmission line (short, medium, or long) the user has assigned based on the length of the transmission line. The range for both the voltage regulation and transmission line efficiency must be within the range of $0 \%$ to $100 \%$ for the transmission line to be applicable, wherein $0 \%$ is the ideal voltage regulation and $100 \%$ for the transmission line efficiency.

\section{METHODOLOGY}

MATLAB will be used to simulate the transmission line performance test. Concepts from the signal transmission and transmission line parameters will be applied in the encoding portion.

The program will begin with the user's inputs. The inputs consist of the transmission line length, receiving end voltage, the resistance per unit length, inductance per unit length, receiving power, lagging factor, and frequency. Depending on the transmission line length, the calculation process for the transmission line will be done in correspondence with the type of transmission line recognized by the system because each type of transmission line tackles a different approach when solving for the $\mathrm{ABCD}$ parameter, which is essential for the calculation of the voltage regulation and transmission line efficiency. The voltage regulation and transmission line efficiency output values will be shown at the end of the program with a short comment on whether the parameters input by the user is applicable or not. Other output parameters, namely the No Load Receiving End Voltage, No Load Sending End Current, and Sending End Power Factor(p.f.) will also be shown in the output. Fig. 1 shows the summarized flow of the program. 


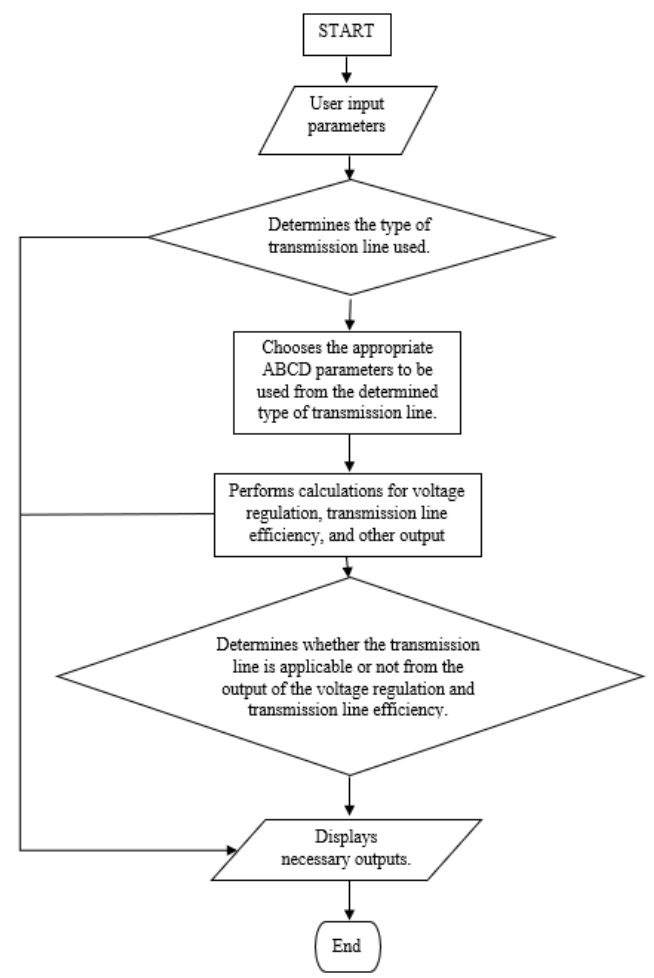

Figure 1: Program Flow

\section{REVIEW OF RELATED LITERATURE}

Like any other electrical system, the transmission lines will have some power losses and voltage drops during transmission. Hence, the transmission line performance test or assessment is done to determine how well the transmitted signal is sent when received at the receiving end based on the voltage regulation and the transmission line efficiency [17].

Transmission lines have three basic electrical parameters: resistance, conductance, and inductance, distributed uniformly along the line. A typical transmission line has a set of conductors, the component for holding these three electrical parameters, placed in specific distances supported by transmission towers.

Electrical power is transmitted over a transmission line theoretically almost in par with the speed of light $\left(3^{*} 10^{\wedge} 8\right.$ $\mathrm{m} / \mathrm{s}$ ). The wavelength produced by the voltage and current of the power may be determined by the formula shown below

$$
\lambda=\frac{v}{f}
$$

Since the electrical power transmitted is almost the same as the speed of light, the formula may be rewritten

$$
\lambda=\frac{c}{f}
$$

In a two-port network representation of a transmission line, $\mathrm{ABCD}$ parameters are used to describe the characteristics of it $[18,19]$. The block representation of a typical two-port network transmission line is shown in Figure 2:

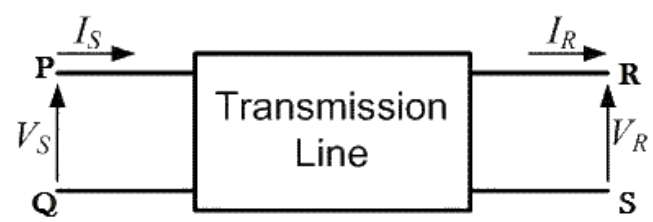

Figure 2: Two-Port Network Transmission Line

Wherein,

$V_{S}=$ supply end voltage

$I_{S}=$ supply end current

$V_{R}=$ received end voltage

$I_{R}=$ received end current

Depending on the length of the transmission line, the ABCD parameters will vary [20]. There are three types of transmission lines: (1) short transmission lines, (2) medium transmission lines, and (3) long transmission lines. The $\mathrm{ABCD}$ parameters are essential for determining the efficiency of the transmission line in order to prevent corona discharge and/or Ferranti effect. Corona discharge is the discharge of electricity caused by the voltage gradient exceeding the critical value because of the "ionization of the surrounding air by high voltage" [21]. Ferranti effect is when the voltage at the receiving end is greater than the sending end voltage due to light load, charging current on the line, or an open circuit at the receiving end [22].

The general equation for acquiring the $\mathrm{ABCD}$ parameters for the transmission line is:

$$
\begin{aligned}
& V_{S}=A V_{r}+B I_{r} \\
& I_{S}=C V_{r}+D I_{r}
\end{aligned}
$$

In order to determine the $\mathrm{ABCD}$ parameters of a transmission line, the short-circuited condition and open-circuited condition of the receiving end are taken into consideration. In the short-circuit condition, the receiving end is shorted, resulting in $V_{R}$ equal to zero [23]. The block representation of a shorted transmission line case is shown in Figure 3.

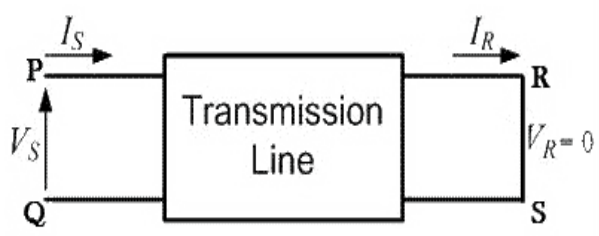

Figure 3: Shorted Two-Port Network Transmission Line

Applying the condition of " $V_{R}=0$ " in the general equation of " $V$ ", the equation can be rewritten as:

$$
\begin{aligned}
& V_{R}=A * 0+B * I_{R} \\
& V_{R}=B I_{R} \quad B=\frac{V_{S}}{I_{R}}
\end{aligned}
$$

During the short-circuited condition, the " $\mathrm{B}$ " parameter is treated resistive in nature because of it being the ratio of the sending end voltage and receiving end current. 
Aaron Don M. Africa et al., International Journal of Emerging Trends in Engineering Research, 8(9), September 2020, 5265 - 5272

For the " $I_{S}$ " case, the formula can be rewritten for the " $V_{R}=0$ " condition as:

$$
\begin{gathered}
I_{S}=C * 0+D * I_{R} \\
I_{S}=D I_{R} \\
\mathrm{D}=\frac{I_{S}}{I_{R}}
\end{gathered}
$$

The "D" parameter then represents the ratio of the sending end current over the receiving end current.

For the open-circuited condition, the $I_{R}$ is equivalent to zero. The two-port open condition transmission line model is shown in Figure 4.

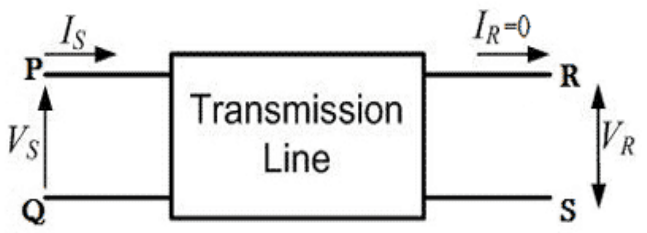

Figure 4: Opened Two-Port Transmission Line

When " $I_{R}=0$ " condition is applied on the general equation of $V_{S}$, the formula may be rewritten as:

$V_{S}=A * V_{R}+B * 0$

$V_{S}=A V_{R}$

$\mathrm{A}=\frac{V_{S}}{V_{R}}$

The "A" parameter represents the ratio sending end voltage and the receiving end voltage.

For the " $I_{R}=0$ " condition on the $I_{S}$ general equation, the formula may be rewritten as:

$I_{S}=C * V_{S}+D * 0$

$I_{S}=C V_{S}$

$\mathrm{C}=\frac{I_{S}}{V_{S}}$

The "C" parameter is conductive (unit in siemens or $\mathrm{S}$ ) in nature for the open-circuited condition shows the composition of the summarized parameter representation, specification, and unit of the ABCD parameters of a transmission line. This is shown in Table 1.

Table 1: Summarized ABCD Parameter Specifications

\begin{tabular}{|l|l|l|}
\hline Parameter & Specification & Unit \\
\hline $\mathrm{A}=\frac{V_{S}}{V_{R}}$ & Voltage Ratio & unitless \\
\hline $\mathrm{B}=\frac{V_{S}}{I_{R}}$ & Short Circuit Resistance & $\Omega$ \\
\hline $\mathrm{C}=\frac{I_{S}}{V_{S}}$ & Open Circuit Conductance & $\mathrm{S}$ \\
\hline $\mathrm{D}=\frac{I_{S}}{I_{R}}$ & Current Ratio & unitless \\
\hline
\end{tabular}

A transmission line having a length of less than $80 \mathrm{~km}$ or 50 miles is considered as a short transmission line [24,25]. In a short transmission line, the transmission line capacitance is negligible because of the small leakage current and other lumped parameters, namely resistance and inductance, along the transmission line as shown in Figure 5.

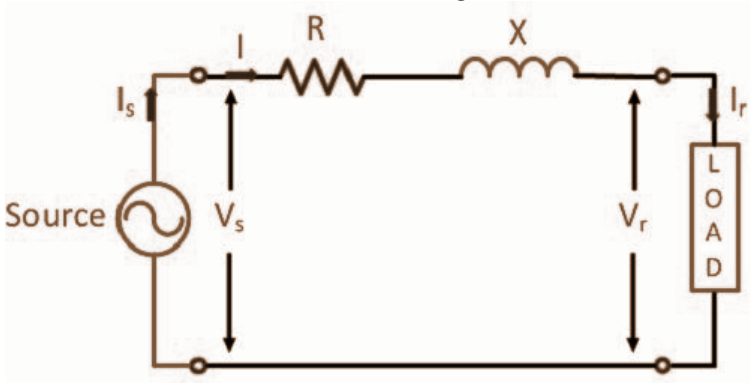

Figure 5: Equivalent Shorted Circuit Model for Short Transmission Line

The ABCD constants of a short transmission line are expressed as:

$$
\begin{aligned}
& A=1 \\
& B=Z \\
& C=0 \\
& D=1
\end{aligned}
$$

The medium transmission line is categorized as such when the transmission line length is within the range of $80 \mathrm{~km}$ to 250 $\mathrm{km}$. The voltage transmission ability for this type of transmission line ranges from $20 \mathrm{kV}$ to $100 \mathrm{kV}$. Compared to the shorted transmission line, the admittance plays a significant role in the analysis portion. When analyzing for the medium transmission line performance, there are three methods available: The Nominal Pi Method, the Nominal T Method, and the End Condenser Method [26].

\begin{tabular}{|c|c|c|c|}
\hline & Short & Medium & Long \\
\hline Length of Wire & $\underset{\mathrm{mi})}{<80 \mathrm{~km}}(\mathbf{5 0}$ & $\begin{array}{c}81 \mathrm{~km}(50 \mathrm{mi}) \\
\text { to } 249 \mathrm{~km}(155 \\
\mathrm{mi})\end{array}$ & $\begin{array}{c}>250 \mathrm{~km}(155 \\
\mathrm{mi})\end{array}$ \\
\hline $\begin{array}{l}\text { Operational } \\
\text { Voltage }\end{array}$ & $<69 \mathrm{kV}$ & $\begin{array}{c}69 \mathrm{kV} \text { to } 133 \\
\mathrm{kV}\end{array}$ & $>133 \mathrm{kV}$ \\
\hline $\begin{array}{l}\text { Capacitance } \\
\text { Effect Taken in } \\
\text { Consideration? }\end{array}$ & No. & Yes. & Yes. \\
\hline Comment & $\begin{array}{l}\text { Only } \\
\text { resistance and } \\
\text { inductance are } \\
\text { taken in } \\
\text { consideration } \\
\text { since } \\
\text { capacitance is } \\
\text { negligible. }\end{array}$ & $\begin{array}{l}\text { Distributed } \\
\text { capacitance } \\
\text { form is used } \\
\text { for calculation } \\
\text { purposes. }\end{array}$ & $\begin{array}{l}\text { Line constants } \\
\text { are considered } \\
\text { as distributed } \\
\text { over the length } \\
\text { of the line. }\end{array}$ \\
\hline
\end{tabular}

Table 2 shows the summarized characteristics of each transmission line based on the length of the transmission line and the operational voltage the transmission line can effectively propagate with brief comments. This is shown in Table 2.

Table 2: Summary of Transmission Line Classifications 
Aaron Don M. Africa et al., International Journal of Emerging Trends in Engineering Research, 8(9), September 2020, 5265 - 5272

The voltage regulation for shorted line condition is the change in voltage at the receiving end when at full load with a given removed power factor and the voltage at the sending end being constant.

\section{THEORETICAL CONSIDERATIONS}

The transmission line performance test for the MATLAB simulation will assume the transmission line to be lossless, meaning the signal will not be damaged or lost during transmission. The inputs asked by the program will initially be in line form, but the process will convert the necessary input parameter, namely the Receiving End Voltage, in its phase form. For the medium transmission line ABCD parameter calculation portion, nominal $\mathrm{T}$ method will be used. The system for the transmission line will assume a balanced three-phase Wye-connection load for the receiving end outputs. At the receiver end for the MATLAB simulation [27], the received power on the load will be $80 \%$ of receiving power input or assigned by the user.

\section{DATA AND RESULTS}

Figures 6 and 7 shows the simulation commands.

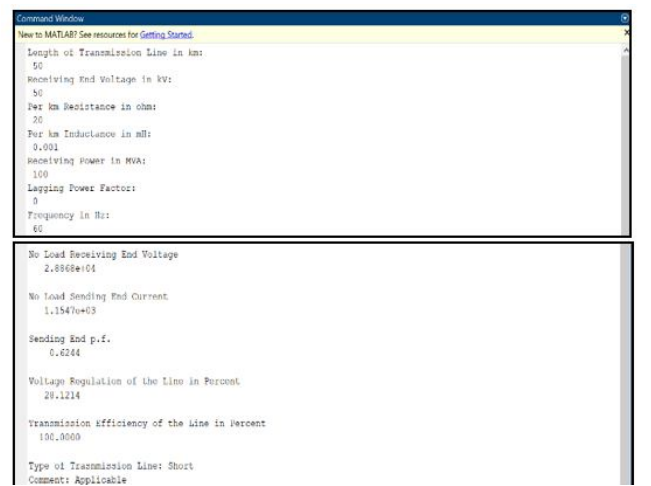

Figure 6: MATLAB Input (Top) and Output (Bottom) Values for Short Transmission Line

Tables 3 and 4 shows the iteration test.

Table 3: Ten Iterations of Short Transmission Line Performance Test (length $=50 ; \mathrm{V} 1=50 ; \mathrm{S}=100 ;$ lagging power factor $=0$;

\begin{tabular}{|c|c|c|c|c|c|c|c|}
\hline length & $\mathbf{V 1}$ & $\mathbf{R}$ & $\mathbf{L}$ & $\mathbf{S}$ & $\mathbf{V R}$ & $\mathbf{E F F}$ & $\mathbf{Y} / \mathbf{~}$ \\
\hline 50 & 50 & 100 & 100 & 100 & $>100 \%$ & $20 \%$ & $\mathrm{~N}$ \\
\hline 50 & 50 & 1000 & 1 & 100 & $>100 \%$ & $2 \%$ & $\mathrm{~N}$ \\
\hline 50 & 50 & 10 & 10 & 100 & $>100 \%$ & $>100 \%$ & $\mathrm{~N}$ \\
\hline 50 & 50 & 10 & 1 & 100 & $79.90 \%$ & $>100 \%$ & $\mathrm{~N}$ \\
\hline 50 & 50 & 50 & 1 & 100 & $>100 \%$ & $40 \%$ & $\mathrm{~N}$ \\
\hline 50 & 50 & 80 & 1 & 100 & $>100 \%$ & $25 \%$ & $\mathrm{~N}$ \\
\hline 50 & 50 & 20 & 1 & 100 & $92.78 \%$ & $100 \%$ & $\mathrm{Y}$ \\
\hline 50 & 50 & 20 & 0.1 & 100 & $34.03 \%$ & $100 \%$ & $\mathrm{Y}$ \\
\hline 50 & 50 & 20 & 0.01 & 100 & $28.65 \%$ & $100 \%$ & $\mathrm{Y}$ \\
\hline 50 & 50 & 20 & 0.001 & 100 & $28.12 \%$ & $100 \%$ & $\mathrm{Y}$ \\
\hline
\end{tabular}

where

length $=$ length of transmission line $(\mathrm{km})$

$\mathrm{V} 1=$ receiving end voltage $(\mathrm{kV})$

$\mathrm{R}=$ resistance $(\mathrm{ohm} / \mathrm{km})$

$\mathrm{L} \quad=$ inductance $(\mathrm{mH} / \mathrm{km})$

$\mathrm{S} \quad=$ receiving power $(\mathrm{MVA})$

$\mathrm{VR}=$ voltage regulation

$\mathrm{EFF}=$ transmission line efficiency

$\mathrm{Y} / \mathrm{N}=$ applicable/not applicable

Table 4: Ten Iterations of Short Transmission Line Performance Test (length $=20 ; \mathrm{V} 1=70 ; \mathrm{S}=70$; lagging power factor $=0.8$; frequency $=50 \mathrm{~Hz}$ )

\begin{tabular}{|c|c|c|c|c|c|c|c|}
\hline length & $\mathbf{V 1}$ & $\mathbf{R}$ & $\mathbf{L}$ & $\mathbf{S}$ & $\mathbf{V R}$ & $\mathbf{E F F}$ & $\mathbf{Y} / \mathbf{N}$ \\
\hline 20 & 70 & 10 & 10 & 70 & $76.97 \%$ & $84.85 \%$ & $\mathrm{Y}$ \\
\hline 20 & 70 & 10 & 1 & 70 & $16.82 \%$ & $84.85 \%$ & $\mathrm{Y}$ \\
\hline 20 & 70 & 5 & 1 & 70 & $11.14 \%$ & $91.80 \%$ & $\mathrm{Y}$ \\
\hline 20 & 70 & 1 & 1 & 70 & $6.72 \%$ & $98.25 \%$ & $\mathrm{Y}$ \\
\hline 20 & 70 & 0.05 & 1 & 70 & $5.68 \%$ & $99.91 \%$ & $\mathrm{Y}$ \\
\hline 20 & 70 & 0.001 & 1 & 70 & $5.63 \%$ & $100 \%$ & $\mathrm{Y}$ \\
\hline 20 & 70 & 0.001 & 0.1 & 70 & $.0 .54 \%$ & $100 \%$ & $\mathrm{Y}$ \\
\hline 20 & 70 & 0.001 & 0.01 & 70 & $0.06 \%$ & $100 \%$ & $\mathrm{Y}$ \\
\hline 20 & 70 & 0.001 & 0.001 & 70 & $0.01 \%$ & $100 \%$ & $\mathrm{Y}$ \\
\hline 20 & 70 & 0.001 & 0.0001 & 70 & $0 \%$ & $100 \%$ & $\mathrm{Y}$ \\
\hline
\end{tabular}

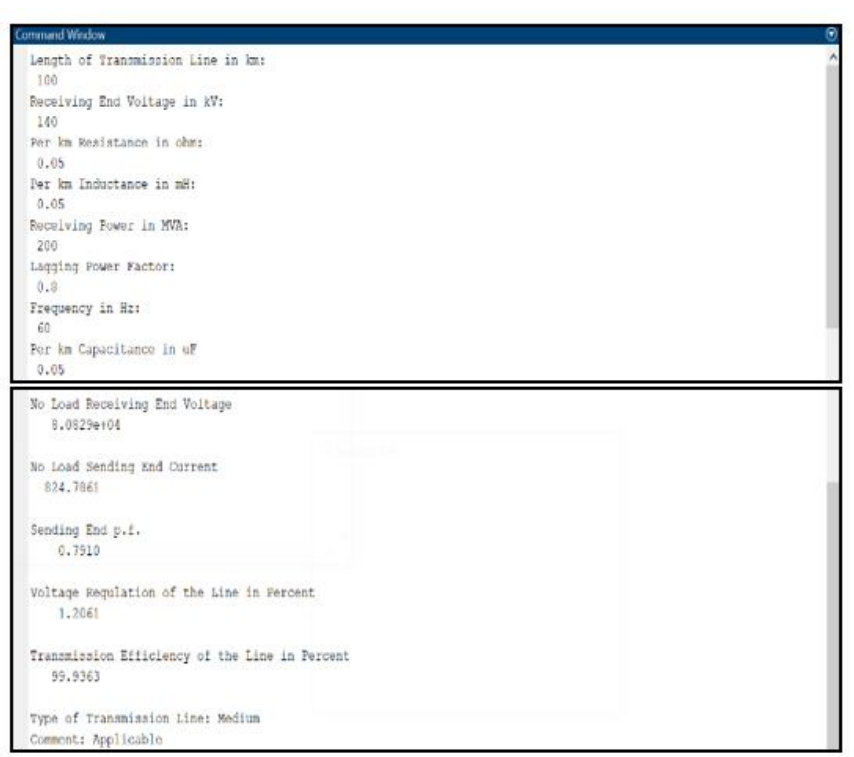

Figure 7: MATLAB Input (Top) and Output (Bottom) Values for Medium Transmission Line

\section{ANALYSIS OF DATA}

The simulation for the transmission line performance test is observed to have the passive components to greatly affect the voltage regulation and transmission line efficiency [28]. 
When the resistance values are varied, both the voltage regulation and transmission line efficiency significantly change. The higher the resistance, the greater the voltage regulation and transmission line efficiency to produce errors, wherein the voltage regulation would increase even greater than $100 \%$ and the transmission line efficiency would lower near $0 \%$ [29]. When the resistance component is decreased, both the voltage regulation and transmission line efficiency would approach the ideal outputs ( $0 \%$ for voltage regulation and $100 \%$ for transmission line efficiency); however, the transmission line efficiency is the parameter most affected by the resistance. Even though the transmission line efficiency will approach $100 \%$ as the resistance lowers, the output could go over $100 \%$, which is not also the ideal output for the transmission line efficiency. When the transmission line efficiency exceeds $100 \%$, a possible reason is that the load at the receiving end might have its own power, even before the transmitted power at the sending end is sent; therefore, the transmitted power must be lowered close to $100 \%$ by varying the resistance parameter of the transmission line.

The inductive and capacitive reactive components of the transmission line adjust the voltage regulation, wherein the lower the value of the reactive components, the better the voltage regulation as it approaches $0 \%$. As observed from Table 3 to Table 4 , the reactive components have no effects on the transmission line efficiency, so in order to obtain a proper working transmission line near the ideal performance values, the resistance is first tampered with, then the reactive components after.

The receiving end voltage and the receiving power have their own limits when attaining the voltage regulation and the transmission line efficiency at a specific length of the transmission. As observed in Tables 3, the receiving voltage at 50 and receiving power at 100 has a limit for the voltage regulation portion at $28 \%$. In Table 4 , the receiving voltage at 70 and receiving power at 70 can produce the ideal voltage regulation. For both Table 3 and Table 4, the receiving voltage and receiving power is observed to have a great contribution in the effect of the transmission line's voltage regulation and line efficiency. When the receiving voltage or power is increased or decreased, the resistance is the parameter needed to vary to adjust both the voltage regulation and line efficiency to their respective closest ideal state because the resistance parameter greatly affects both the voltage regulation and line efficiency. The inductance, for the short transmission line case, will be varied too for the voltage regulation only after the resistance is adjusted to the proper amount for the line efficiency. In the medium and long transmission line case, either the inductance or the capacitance may be adjusted after the resistance parameter is adjusted.

\section{CONCLUSION}

The transmission line performance test is successful in its simulations for calculating the essential output parameters, commenting on the type of transmission line, and determining whether the input parameters assigned by the user is applicable for real-life uses.

Depending on the length of the transmission line, the program performed its designated $\mathrm{ABCD}$ parameter process. For the short transmission line condition $(>0 \mathrm{~km}$ to $<80 \mathrm{~km}$ ), the transmission line theoretically has the capacitance considered to be negligible, meaning only the resistance and inductance are varied after considering the receiving voltage and receiving power input for a better voltage regulation and transmission line efficiency. Starting at the medium transmission line $(80 \mathrm{~km}$ to $<250 \mathrm{~km})$ until the long transmission line ( $250 \mathrm{~km}$ above), the capacitance is taken into consideration for determining the voltage regulation and the transmission line efficiency. The difference between the medium transmission line and the long transmission line is how intricate the calculations are when the capacitance is taken into consideration. The long transmission line requires complex calculations because of the number of lumped distributed parameters along the transmission line. The longer the length of the transmission line, the more likely for the power to drop, resulting in the voltage regulation to rise and the line efficiency to drop.

\section{RECOMMENDATIONS}

The transmission line performance test performed by this program works best with the conditions stated by the limitations: (1) there is no outside interference to disrupt the transmission, (2) the circuit configuration to be in Wye-connection load, and (3) a power factor of the received end power to be $80 \%$ of the receiving power, so there are some recommendations to further improve the program and add some additional features so that the program will not be limited only to the Wye-connection case.

An alternative concept that may be used in determining the performance of the transmission line is the SWR and the reflection coefficient. The SWR can determine if the transmission line is working properly and efficiently by sending an incident signal and observing how much of the reflected signal is received or how much of the SWR is increased or decreased. The reflection coefficient can determine if the transmission line may have an open or short.

An extra feature that could be added is to take into account the delta connection calculations, because there are connections in real-life that use delta connection and/or mixed delta-wye/wye-delta connections. The algorithm using the ideal voltage regulation and transmission line efficiency as the basis for determining the passive parameter values or the receiving voltage and receiving power is another feature that could be added in the program. The first case is that given a designated receiving voltage and receiving power with specific lagging power factor and frequency, the program will use the ideal voltage regulation and transmission line efficiency to perform reverse calculations to obtain the minimum passive parameters to satisfy the ideal voltage regulation and transmission line efficiency condition. The second case follows the same idea as the first case, but the 
constant or designated values will be the passive parameters instead of the receiving voltage and receiving power, wherein the outcome will be the receiving voltage and receiving power themselves to satisfy the ideal voltage regulation and transmission line efficiency.

\section{REFERENCES}

[1] J. Santiago and M. C. Tavares, "Analysis of halfwavelength transmission line under critical balanced faults: Voltage response and overvoltage mitigation procedure," Electric Power Systems Research, vol. 166, pp. 99-111, Jan. 2019.

[2] E. F. Rosales, "Electricity demand to reach all-time high this year-NGCP: Elijah Felice Rosales," BusinessMirror, 27-Mar-2019.

[3] E. Perez, J. Espinosa, and D. Aranguren, "On the development of dynamic stroke density for transmission line for power system operational applications," International Journal of Electrical Power \& Energy Systems, vol. 116, p. 105527, Mar. 2020.

[4] X. Fu and H.-N. Li, "Dynamic analysis of transmission tower-line system subjected to wind and rain loads," Journal of Wind Engineering and Industrial Aerodynamics, vol. 157, pp. 95-103, Oct. 2016.

[5] A. Baloi, A. Pana, and F. Molnar-Matei, "Advantages of Using MatLab Simulink in Laboratory Lessons on Operating Conditions of Overhead Power Lines," Procedia -Social and Behavioral Sciences, vol. 191, pp. 179-184, Jun. 2015.

[6] J. M. M. Beça, J. M. B. Bezerra, J. E. M. Quijada, L. V. G. Pessoa, and R. R. D. Silva, "A Performance Analysis of Uprating Techniques on a $230 \mathrm{KV}$ Overhead Transmission Line," Smart Innovation, Systems and Technologies, vol. 140, pp. 3-13, 2019.

[7] A. Ukil, "Theoretical analysis of tuned HVAC line for low loss long distance bulk power transmission," International Journal of Electrical Power \& Energy Systems, vol. 73, pp. 433-437, Dec. 2015.

[8] F. Alminhana, M. Mason, and F. Albermani, "A compact nonlinear dynamic analysis technique for transmission line cascades," Engineering Structures, vol. 158, pp. 164-174, Mar. 2018.

[9] J. D. Sakala, J. S. J. Daka, D. Setlhaolo, and A. P. Malichi, "Transmission Line Adapted Analytical Power Charts Solution," Journal of The Institution of Engineers (India): Series B, vol. 98, no. 4, pp. 433-440, Aug. 2017.

[10] R. Balagopal, A. Ramaswamy, G. Palani, and N. P. Rao, "Simplified bolted connection model for analysis of transmission line towers," Structures, vol. 27, pp. 2114-2125, Oct. 2020.

[11] M. Löschenbrand, "A transmission expansion model for dynamic operation of flexible demand," International Journal of Electrical Power \& Energy Systems, vol. 124,Jan. 2021.

[12] S. J. Chuang, C. M. Hong, and C. H. Chen, "Improvement of integrated transmission line transfer index for power system voltage stability," International Journal of Electrical Power \& Energy Systems, vol. 78, pp. 830-836, Jun. 2016.
[13] P. Stefánsson, A. D. Sæpórsdóttir, and C. M. Hall, "When tourists meet transmission lines: The effects of electric transmission lines on tourism in Iceland," Energy Research \& Social Science, vol. 34, pp. 82-92, Dec. 2017.

[14] A. B. Alhassan, X. Zhang, H. Shen, and H. Xu, "Power transmission line inspection robots: A review, trends and challenges for future research," International Journal of Electrical Power \& Energy Systems, vol. 118, p. 105862 , Jun. 2020.

[15]J. Aghaei, A. Nikoobakht, M. Mardaneh, M. Shafie-Khah, and J. P. Catalão, "Transmission switching, demand response and energy storage systems in an innovative integrated scheme for managing the uncertainty of wind power generation," International Journal of Electrical Power \& Energy Systems, vol. 98, pp. 72-84, Jun. 2018.

[16] B. Li and M. Lv, "A calculation method of transmission line equivalent geometrical parameters based on power-frequency parameters," International Journal of Electrical Power \& Energy Systems, vol. 111, pp. 152-159, Oct. 2019.

[17]Electrical4U, "Performance of Transmission Line,"https://www.electrical4u.com/performance-of-tran smission-line/, 2018.

[18] C. Alexander and M. Sadiku, "Fundamentals of Electric Circuits Fourth Edition," McGraw-Hill, 2009.

[19] E. Panov, E. Barudov and M. Ivanova, "Exploration of the Two-Port Parameters of Autotransformer Discrete Alternating Voltage Regulators," 2019 11th Electrical Engineering Faculty Conference (BulEF), Varna, Bulgaria, pp. 1-4, 2019.

[20] Electrical4U, “ABCD Parameters of Transmission Line: https://www.electrical4u.com/abcd-parameters-of-trans mission-line/, 2020.

[21] "Corona Discharge," THEFREEDICTIONARY: https://encyclopedia2.thefreedictionary.com/Corona+Dis charge, 2020.

[22] "Ferranti Effect," Circuit globe:https://circuitglobe.com/ferranti-effect.html. 2020

[23] J. Colqui, S. Kurokawa, and J. Pissolato, "An alternative procedure to obtain the ABCD matrix of multiphase transmission lines: Validation and applications," Electric Power Systems Research. vol. 180, Mar. 2020.

[24] "Short Transmission Line," Circuit globe: https://circuitglobe.com/short-transmission-line.html\#: : text=A\%20transmission $\% 20$ line $\% 20$ having\%20its,lump ed\%20in\%20the\%20transmission\%20line, 2020.

[25] C. M. Pacis, G. R. Maramba and C. E. Calamayan, "Experimental design and performance for medium transmission lines using TQ NE9080 line simulator," TENCON 2017 - 2017 IEEE Region 10 Conference, Penang, pp. 2347-2352, 2017.

[26] "Medium-length Transmission Line," Chegg Study: https://www.chegg.com/homework-help/definitions/med ium-length-transmission-line-4\#: :text=Medium\%20len gth\%20transmission\%20lines\%20are, 20\%20KV\%20to $\% 20100 \% 20 \mathrm{KV} . \&$ text=Nominal $\% 20 \%$ CE\%A0\%20met hod $\% 3$ A,is $\% 20$ the $\% 20$ nominal $\% 20 \%$ CE\%A0\%20circui t. 2020 . 
Aaron Don M. Africa et al., International Journal of Emerging Trends in Engineering Research, 8(9), September 2020, 5265 - 5272

[27] Matlab.

https://www.mathworks.com/products/matlab.html.2020

[28] A. Africa and J. Lao, "Development of a wave propagation model in a lossy transmission line," International Journal of Emerging Trends in Engineering Research, vol. 8, no. 7, pp. 3351-3357, 2020.

[29]M. Said, M. Misran, Z. Zakaria, M. Zin and L. Muhammad, "Design of a circularly polarized antenna at $2.45 \mathrm{ghz}$ with harmonic suppression for material characterization," International Journal of Emerging Trends in Engineering Research, vol. 8, no. 8, pp. 4420-4425, 2020. 